Gynäkologe 2009 · 42:245-246

DOI 10.1007/s00129-008-2307-5

Online publiziert: 3. April 2009

(c) Springer Medizin Verlag 2009

\author{
R. Kürzl·K. Friese \\ Frauenklinik der LMU München, Campus Innenstadt, München
}

\section{Erkrankungen der Vulva}

denn habe, so sind die Antworten fast immer gleich: es handle sich um wiederkehrende Pilzinfektionen oder um altersbedingte Hautveränderungen wegen Östrogenmangels. Die Beiträge in diesem Heft sollen zeigen, dass diese Sichtweise in Diagnostik und Therapie viel zu kurz greift.

\section{Weites Spektrum der Differenzialdiagnose}

Es war Absicht der Herausgeber, das verkannte oder unbekannte, weite Spektrum der Differenzialdiagnose der letztlich monotonen Symptomatik von Juckreiz, Brennen und Gefühl des Wundseins zur Darstellung zu bringen.

Infektionserkrankungen der Vulva umfassen weit mehr an Krankheiten als nur die Miterkrankung der Vulva bei einer Soorkolpitis (Beitrag Mylonas und Petersen). Die klinischen Bilder zeigen aber immer wieder Überschneidungen mit denen nichtmaligner Erkrankungen der Vulva (Beitrag Kürzl). Insbesondere, wenn die Behandlung unter der Diagnose Infektion nicht zum gewünschten Erfolg führt, sind sie differenzialdiagnostisch zu berücksichtigen. Der Lichen sclerosus ist mit Abstand die häufigste Erkrankung der Vulvahaut und sollte deshalb auch von Gynäkologen adäquat behandelt werden können. Andere nichtmaligne Hauterkrankungen treten an der Vulva zwar nur selten auf, aber das Wissen, dass es solche Affektionen auch an der Vulva geben kann, sollte zumindest dafür sensibilisieren, eine betroffene Frau, wenn man mit der Diagnostik selbst nicht mehr weiterkommt, zum spezialisierten Gynäkologen zu überweisen, der auch mit Dermatologen zusammenarbeitet. Eine solche interdisziplinäre Kooperation sollte sich im Allgemeinen zum Wohle der Patientin auswirken.

Die Differenzialdiagnose muss aber auch präinvasive Erkrankungen und schließlich das Vulvakarzinom berücksichtigen. Zu Ersteren zählen die vulväre intraepitheliale Neoplasie (VIN) und der nungslos die Veränderungen der Anatomie und der Feinstruktur der Haut erkennen, hinter denen sich fast immer quälende Beschwerden der betroffenen Frauen verbergen und wegen derer sie therapeutische Hilfe beim Arzt suchen.

\section{Die Vulva, medizinisch häufig eine Terra incognita der Hautoberfläche}

Die Hilfe ist für die Frauen nicht immer leicht $\mathrm{zu}$ finden, denn die verordneten Externa lindern meist nur für kurze Zeit die Beschwerden, aber nicht durchgreifend und dauerhaft. Fragt man die Patientin, die ihre Plastiktüte mit zahlreichen angebrochenen Tuben vorzeigt, ob man ihr mitgeteilt hätte, welche Krankheit sie

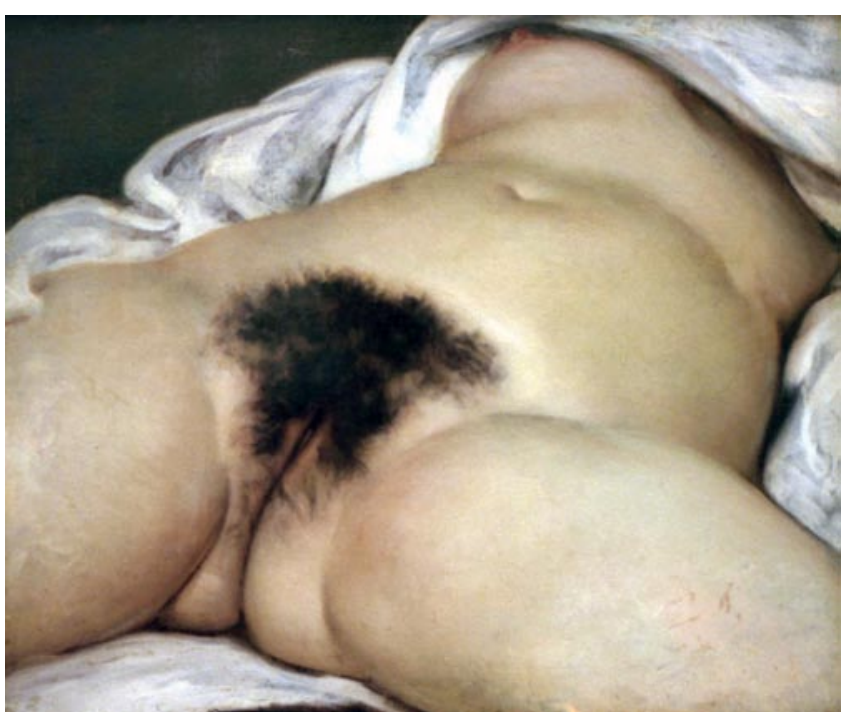
(Gustave Courbet, 1866) 
M. Paget der Vulva (Beitrag Kürzl). Das Vulvakarzinom (Beitrag Hantschmann) kann unter bestimmten Bedingungen lokal und regionär eingeschränkt behandelt werden, d. h. radikale lokale Exzision statt radikaler Vulvektomie und Sentinellymph-node-Verfahren (SLN) statt kompletter inguinofemoraler Lymphonodektomie mit ihrer lästigen Morbidität.

Der abschließende Beitrag von Beck und Freundl thematisiert die weibliche Beschneidung, der immer noch kleine Mädchen vorwiegend in afrikanischen Regionen unterzogen werden. Während das Vulvakarzinom zunehmend möglichst organerhaltend behandelt werden kann, beraubt die Beschneidung gesunde Kinder ihrer Schamlippen und ihrer Klitoris aus Gründen, die in westlichen Ländern als nicht nachvollziehbar gelten. Früher oder später können nicht zuletzt Erkrankungen der Vulva als Sekundärfolgen nach einer Beschneidung entstehen.

( Erkrankungen der Vulva sollten ihren Status als „Mauerblümchen” verlieren

Die Herausgeber des Themenhefts danken den Autoren für ihre Arbeit und der Redaktion für ihre Bereitschaft, die zahl- reichen Abbildungen zum Abdruck zu akzeptieren. Damit ist es möglich geworden, dem Leser das breite Spektrum der Erkrankungen der Vulva sichtbar vor Augen zu führen in der Hoffnung, die Differenzialdiagnose bei Hautveränderungen der Vulva für die Kolleginnen und Kollegen wesentlich zu erweitern. Die Erkrankungen der Vulva müssen aus ihrem Status als „Mauerblümchen” herausgeholt werden; denn oft hat die Patientin den Eindruck, kein Arzt wolle sie mit ihren Beschwerden haben, nicht der Frauenarzt und nicht der Hautarzt. Um diesem Eindruck entgegenzuwirken, sollte großzügig die interdisziplinäre Zusammenarbeit angestrebt werden, insbesondere im Rahmen von Spezialsprechstunden für Frauen mit Vulvaerkrankungen. Auf internationaler und europäischer Ebene gibt es dazu wissenschaftliche Gesellschaften: die International Society for the Study of Vulvovaginal Disease (ISSVD) und das European College for the Study of Vulval Disease (ECSVD). Sie haben nicht nur Gynäkologen und Dermatologen als Mitglieder, sondern als dritten Partner auch die Pathologen. Das ECSVD wird seine nächste Tagung mit Fortbildungskurs im September 2010 in München (ecsvd.eu) abhalten. Wenn Sie durch die Beiträge in diesem Heft neugierig geworden sind und zu Erkrankungen der Vulva noch mehr erfahren wollen, kann die Teilnahme an dieser Tagung nur nachdrücklich empfohlen werden.

Mit freundlichen kollegialen Grüßen

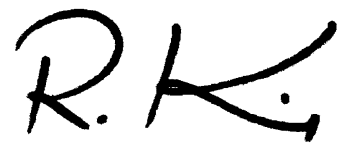

Professor Dr. R. Kürzl

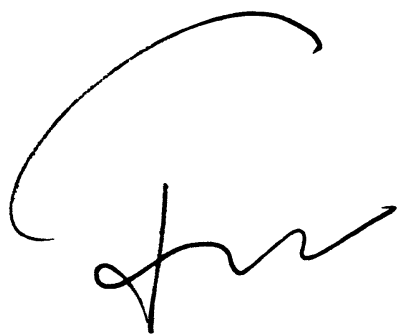

Professor Dr. K. Friese

\section{Korrespondenzadresse}

Prof. Dr. R. Kürzl

Frauenklinik der LMU München,

Campus Innenstadt

Maistr. 11, 80337 München

rainer.kuerzl@med.uni-muenchen.de
Exklusiv für Abonnenten :

\section{Nutzen Sie das Online-Archiv von Der Gynäkologe}

Ihre Vorteile:

- Komfortable und schnelle Recherche nach Themen, Autoren, Suchbegriffen

- Ob unterwegs oder am eigenen PC: Zugriff überall und jederzeit

- Online First: Lesen Sie die aktuellsten Beiträge schon vor Erscheinen des gedruckten Heftes online

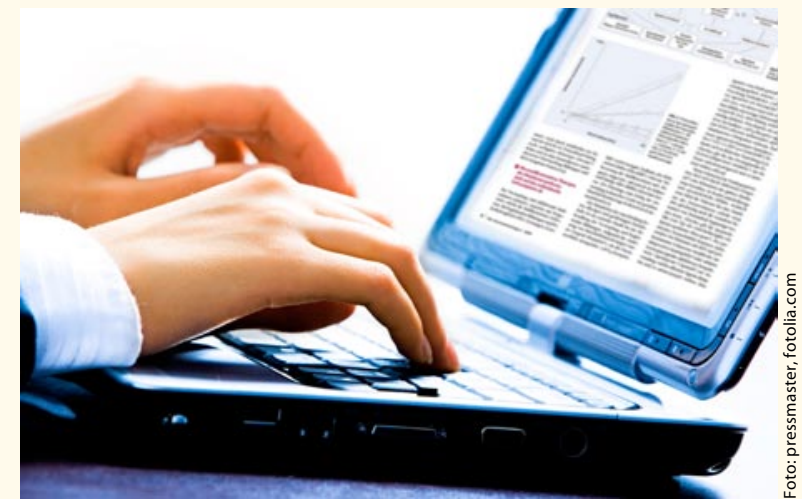

